\title{
The structure of the pulsar magnetosphere via particle simulation with GRAPE
}

\author{
Shinpei Shibata \\ Department of Physics, Yamagata University, Kojirakwa, Yamagata, Japan \\ email: shibata@sci.kj.yamagata-u.ac.jp
}

\begin{abstract}
It is shown with a particle simulation that the outer gap can be reproduced under a few simple assumptions. The simulation includes just the first principles, namely Maxwell's equations, a relativistic equation of motion with radiation drag, and electron-positron pair creation. We also suggest that the Y-point (the open-close boundary in the equator) is likely to be a place of heating and acceleration of plasma, and therefore it would cause high-energy emission. The dead zone along the separatrix of the oppositely directed current is found in the middle latitude region, which separates the outer gap and the polar cap accelerators.
\end{abstract}

Keywords. magnetosphere, plasmas, gamma-ray

\section{Introduction}

The outer gaps of the pulsar magnetosphere were theoretically suggested as a source of high-energy emission by Holloway (1973) around the null surface and by Cheng, Ho \& Ruderman (1976) in view of the topology of the photon path. Recently, Fermi gamma-ray observations favor it as one of the dominant sources (see the review by Saz Parkinson, this volume). In this paper, we intend to examine the reality of the outer gaps via particle simulation.

\section{Particle simulation}

In our particle simulation, we assume axisymmetry and find a steady solution, so that Maxwell's equations becomes Poisson's equations for the electric potential and for the vector potential, which can be solved quickly by using GRAPE, the special-purpose computer for gravitational many-body problems, located at the National Astronomical Observatory of Japan. The plasmas in the magnetosphere are represented by several tens of thousands of super-particles. We iteratively solve the equation of motion for the particles and the electromagnetic field until a steady state is achieved. The calculation domain is three dimensional, in a cube with sides of 60 light-cylinder radii.

We take into account the radiation drag force in the equation of motion. The mass and the charge of the super particles are chosen so that the gyro radii are much smaller than the star. The time step is smaller than the shortest gyro period. Thus we follow any kind of drift motions.

The magnetospheric plasma is supplied in two ways. One is from the stellar surface if there is field-aligned electric field. If the electric field is screened, the emission stops, i.e., the condition of free emission on the stellar surface. The other is due to pair creation. Although conversion from the high energy gamma-ray into pairs should be treated, we simply assume that if the field-aligned electric field $E_{\|}$at a given point is larger than a critical value $E_{c}$, the pairs are put there in a rate proportional to $\left|E_{\|} / E_{c}\right|$ : on the spot approximation. 


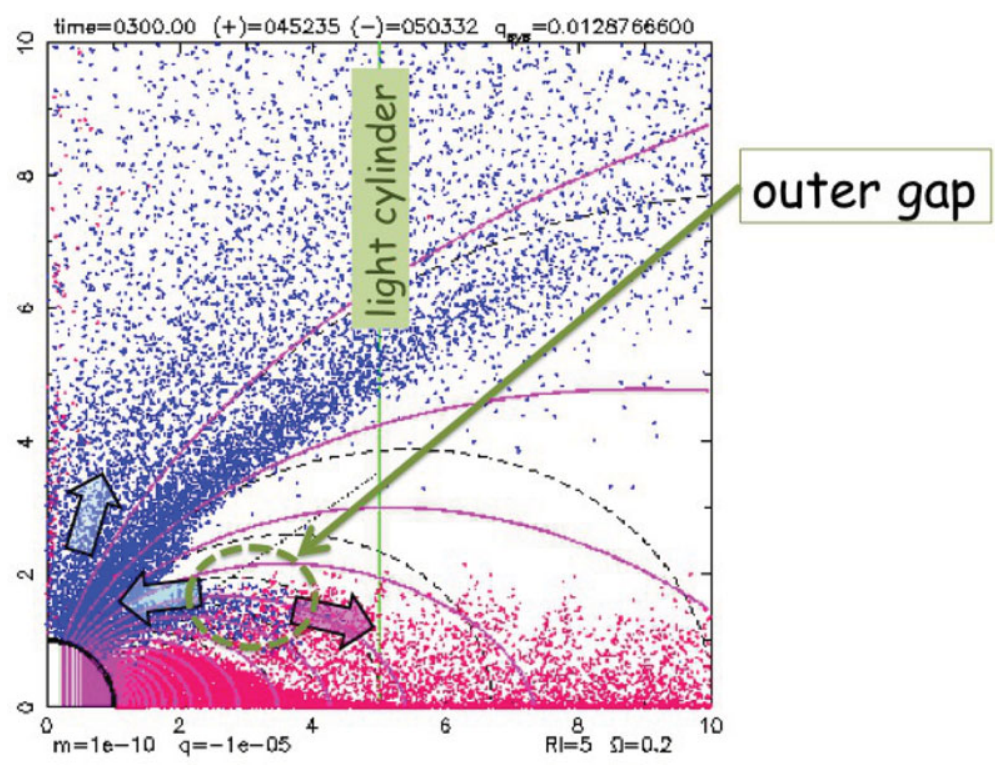

Figure 1. Particle distribution and magnetic field lines (solid curve) in the steady state. The dashed curves indicate dipole field.

Needless to say, the inner boundary for the electric potential provides electromotive force by the rotating magnet. This boundary condition is the source of all the magnetic activity and is strictly satisfied in our simulation because we use the Green function satisfying the boundary condition when we calculate the electric field.

We obtained the steady solution, in which the outer gap is formed and continuously produces electron-position pairs but still have the field-aligned electric field which is slightly larger than $E_{c}$, as shown in Fig. 1 (Wada \& Shibata 2007). The pairs produced in the outer gap are immediately separated by sign of charge; positive particles go out toward the light cylinder while negative particles go back to the star (the polarity depends on direction of the magnetic moment to the rotation).

The gap formation is due to the charge deficiency compared to the Goldreich-Julian density needed to screen the magnetic-field-aligned electric field. This situation takes place because the pulsar has very strong electromotive force and therefore requires high charge density to screen out the field-aligned electric field, while the source of plasma is quite limited. This is relaxed by electron-positron pair creation. The charge deficiency is resolved by pair creation, and at the same time the poloidal current system completes. Thus even for the axisymmetric system, the rotating magnet can release rotational energy. In our simulation, pair creation steadily continues. Pairs can close the gap, so the fieldaligned electric field and pairs can appear intermittently and alternatively.

The positive particles flow out across the closed magnetic field due to radiation drag drift, which is the back reaction of emission of angular momentum by photons. Because the mass is artificially enlarged in super particles, the size of this region is large in the simulation, in the actual magnetosphere flowing-out takes place in a thin current sheet. It is notable that the outer gap causes super-corotation beyond the outer gap (Wada \& Shibata 2011).

The negative charges flowing back to the star charge it up, but this never continues, and emission of particles from the polar caps compensates, so that the system finally becomes in a steady state. 


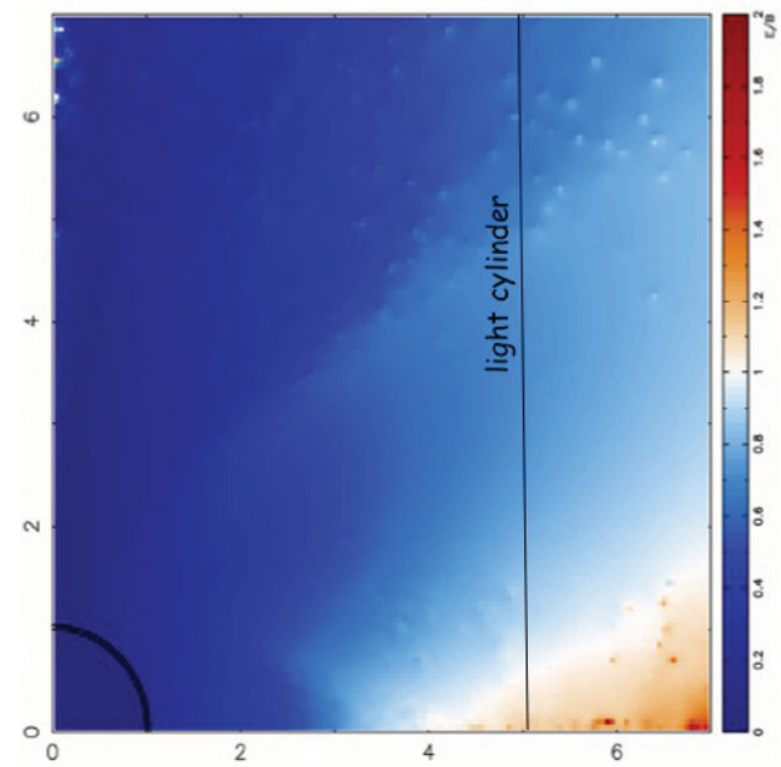

Figure 2. The strength of the electric field. The reddish regions indicate $E>B$.

\section{Y-point}

Since the energy is provided by rotation, the centrifugal force plays a most important role. It is notable that azimuthal velocity at the top of the corotating dead zone approaches the speed of light, and the inertial mass diverges and breaks open the closed magnetic field. This effect is cased by the perpendicular electric field to the magnetic field, $\vec{E}_{\perp}$, causing $\vec{E} \times \vec{B}$-drift motion in azimuthal direction.

It is interesting that the electric field larger than the magnetic field appears in a region around the equatorial neutral sheet beyond the light cylinder, as seen in Fig. 2 (Yuki \& Shibata 2012). This is very much similar to the result of Uzdensky (2004) in his force-free model. The top of the closed field region, and beyond, is the transition region from the closed-open topology which can be one of the most active regions in the magnetosphere. In this region, strong acceleration by the electric field $\vec{E}_{\perp}$ causes a jet outward along the equatorial plane. This is actually due to strong (relativistic) centrifugal force and a particular feature of the pulsar magnetosphere. Although this result is found in our steady solution, the jet could be quasi-steady with magnetic reconnection. We have made a particle-in-cell simulation to find quasi-periodic reconnection derived by centrifugal force at the top of the dead zone (Umizaki \& Shibata 2010). We need further study for this region. The pulse shape from this region is quite difficult to predict because this shape is very sensitive to the dynamics, which are difficult to solve at the moment.

\section{Current neutral dead zone}

A new finding for the global structure is a dead zone in the middle latitudes elongating along a separatrix of the poloidal current(Yuki \& Shibata, 2012). Thus we have two kinds of dead zone. One is the dead zone with closed magnetic field found in classical models. The new dead zone is located above the outer gap, and the poloidal current diminishes and reveres across the dead zone as seen in Fig. 3. The outer gap is sandwiched between the traditional dead zone with closed field region and this new dead zone, which may 


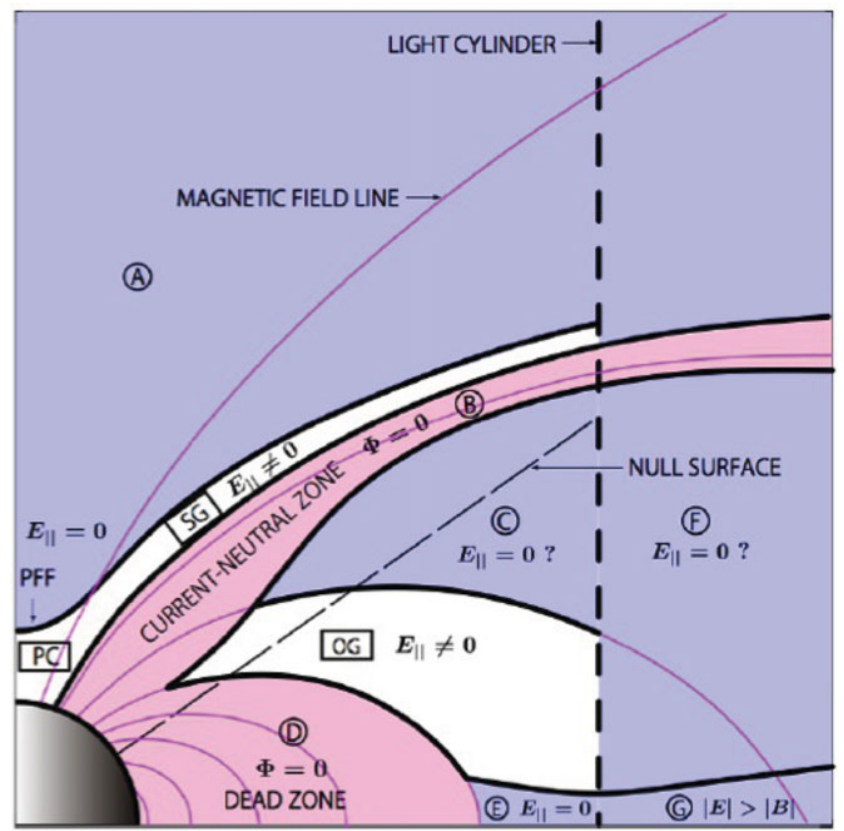

Figure 3. Schematic picture of the axisymmetric magnetosphere found by the particle simulation. PC, SG and OG indicate the polar cap, slot gap and outer gap, respectively.

be called "current neutral dead zone", located in the middle latitudes. The accelerators in the polar caps and the slot gaps are located above the current neutral dead zone. Sometimes the slot gaps are reported to be below the outer gap. However, our simulation suggest that this is wrong and that the slot gaps are above the current neutral dead zone.

\section{Future works}

In future simulations, we need to take the following points in account:

(a) use realistic pair creation processes (not on the spot approximation),

(b) make larger numbers of particle to have a pulsar wind,

(c) use finer simulation particles so that the polar cap structure is resolved.

The computational requirements are the most serious problem. In Fig. 1, one can see that pairs are produced along the rotation axis. These pairs are not produced in the polar cap accelerator but just by fluctuating electric field because the resolution is not enough. If the number of particles were much larger, pair discharge such as obtained in Timokhin (2010) might take place.

\section{References}

Cheng, A., Ruderman, M., \& Sutherland, P., 1976, ApJ, 203,209

Holloway, N. J. 1973, Nat. Phys. Sci., 246,6

Timokhin, A. N. 2010, Mon. Not. R. astron. Soc., 408, 2092

Umizaki M. \& Shibata S. 2010, Pub. Astr. Soc Japan, 62, 131

Uzdensky, D. A., 2004, ApJ, 603, 652

Wada T. \& Shibata S., 2007, Mon. Not. R. astron. Soc., 376, 1460

Wada T. \& Shibata S., 2011, Mon. Not. R. astron. Soc., 418, 612

Yuki S. \& Shibata S., 2012, Pub. Astr Soc. Japan, 64, 43 